\title{
Origen del Nervio Subescapular Inferior, Patrones de Ramifica- ción y su Contribución a la Inervación del Músculo Subescapular
}

\author{
Origin of the Inferior Subscapular Nerve, Branching Patterns \\ and its Contribution to the Subscapular Muscle Innervation
}

Bustamante Aliste, P. A. ${ }^{1,2}$; Sousa Rodrigues, C. F. ${ }^{3}$ \& Olave, E. ${ }^{4}$

\begin{abstract}
BUSTAMANTE ALISTE, P. A.; SOUSA RODRIGUES, C. F. \& OLAVE, E. Origen del nervio Subescapular Inferior, patrones de ramificación y su contribución a la inervación del músculo subescapular. Int. J. Morphol., 38(1):176-181, 2020.

RESUMEN: El nervio subescapular inferior (NSI) inerva parcialmente al músculo subescapular (MSe) e inerva también al músculo redondo mayor (MRM). Diversas publicaciones determinan amplia variación en su origen en el Plexo Braquial (PB), pero existe poca evidencia de estas variaciones y del patrón de inervación del MSe y MRM en individuos latinoamericanos. El propósito de este estudio fue describir el origen del NSI en el PB, determinar número de ramos que le entrega al MSe y los patrones de ramificación. Se utilizaron 30 miembros superiores de individuos adultos, Brasileños; 13 del lado derecho y 17 del izquierdo, fijados en formaldehido al $10 \%$. Se disecaron las regiones axilares para exponer el fascículo posterior del plexo braquial (FPPB) y sus ramos. Se determinó si el origen del NSI era individual o procedía de un tronco común. Se cuantificó el número de ramos para el MSe, estableciendo patrones de ramificación. El NSI y sus ramos se agruparon según su origen y ramificación. En 3 de los casos (10 \%) el NSI procedía de un tronco común con el nervio toracodorsal (NTD), 2 del lado izquierdo (6,6\%) y 1 del derecho (3,3\%); en 27 casos (90\%) procedía del nervio axilar (NAx), 15 del lado izquierdo (50\%) y 12 del derecho (40\%). En ningún caso, el origen fue directo del FPPB. Además, se cuantificó el número de ramos que aportaba a la inervación del MSe, observándose un promedio de 4 ramos (de 1 a 8 ramos) para el MSe. Se identificaron 4 patrones de ramificación del NSI hacia el MSe y el MRM. Tanto el origen como la distribución del NSI presentaron variaciones. Los datos aportados complementarán los conocimientos para la correcta enseñanza, el oportuno diagnóstico y la buena práctica quirúrgica de la zona axilar.
\end{abstract}

PALABRAS CLAVE: Anatomía; Plexo braquial; Nervio subescapular inferior; Músculo subescapular.

\section{INTRODUCCIÓN}

La Terminología Anatómica Internacional (Federative Committee on Anatomical Terminology, 1998) ha descrito de manera inespecífica a un grupo de nervios subescapulares sin distinguir entre uno superior y uno inferior (lo que da indicios de la amplia variación que es posible encontrar en este grupo de nervios). Sin embargo, en la literatura clásica el nervio subescapular inferior (NSI) se describe como un nervio que aporta con la inervación de los fascículos medios e inferiores del músculo subescapular (MSe) y se distingue del nervio para el músculo redondo mayor, que es un nervio independiente, en cuya descripción también alcanzaría los fascículos inferiores del músculo subescapular (Rouviére \& Delmas, 2005). Esto muchas veces puede confundir, puesto que actualmente es más común encontrar la descripción de un nervio subescapular inferior con la función común de inervar los fascículos inferiores del músculo subescapular y como el principal encargado de inervar al músculo redondo mayor (MRM) (Dancker et al., 2015), aunque en este estudio no se desarrollará discusión en relación al nombre utilizado en la literatura. Su origen comúnmente se describe desde los segmentos C6 (Moore $e t$ al., 2013) o C5, C6 y C7, normalmente con un origen directo desde el fascículo posterior del plexo braquial (PB) (Kim et al., 2008). Sin embargo, en otras descripciones clásicas se describen nervios subescapulares superiores e inferiores y un nervio para el músculo redondo mayor como un nervio independiente, con origen diverso, presentación que es menos frecuente (Ballesteros \& Ramirez, 2014).

Existen además diversas publicaciones que determinan amplia variación en el origen del nervio subescapular inferior desde el plexo braquial, describiéndose el origen

\footnotetext{
${ }^{1}$ Departamento de Ciencias Morfológicas, Facultad de Medicina y Ciencia, Universidad San Sebastián, Puerto Montt, Chile

${ }^{2}$ Programa de Magister en Ciencias, mención Morfología, Universidad de La Frontera, Temuco, Chile.

${ }^{3}$ Universidad Estadual de Ciencias da Saude de Alagoas, Maceió, Brasil.

${ }^{4}$ Facultad de Medicina, Universidad de La Frontera, Temuco, Chile.
} 
tanto desde el fascículo posterior, como se ha descrito generalmente, así como desde el nervio radial (NR) o desde el nervio axilar (NAx) (Aggarwal et al., 2009), además de presentar una variable distribución, compartiendo en algunos casos un tronco común junto con el nervio toracodorsal (NTD) (Muthoka et al., 2011). Todo lo anterior será siempre un posible hallazgo quirúrgico debido a la gran variabilidad en las conexiones durante la formación del plexo braquial (Uzun \& Bilgiç, 1999), por lo que también es esperable encontrar variaciones en los ramos que se forman en él. No obstante, existe poca evidencia y escasa descripción de éstas y otras variaciones, de su distribución en la región escapular, y del patrón de inervación para los músculos subescapular y redondo mayor por parte del nervio subescapular inferior en individuos latinoamericanos.

El propósito de este estudio fue describir el origen del nervio subescapular inferior desde el plexo braquial, determinar el número de ramos que le entrega al músculo subescapular, sus patrones de ramificación hacia el mismo y al músculo redondo mayor, para finalmente proponer una clasificación para estas variaciones, con lo que se espera contribuir al entendimiento de la organización y relación de las distintas estructuras ubicadas en la región axilar, y favorecer el quehacer quirúrgico en distintas intervenciones de la misma.

\section{MATERIAL Y MÉTODO}

Se utilizaron 30 miembros superiores de individuos brasileños; 13 del lado derecho y 17 del izquierdo, fijados en formaldehido al $10 \%$, pertenecientes al Laboratorio de Anatomía Humana de la Universidad Estadual de Ciencias da Saude de Alagoas, Maceió, Brasil. En algunos de los casos el miembro superior se encontraba separado del resto del cuerpo, lo que no permitió establecer el sexo del sujeto, por lo que dicho parámetro no pudo ser considerado dentro del análisis de los datos.

Se accedió a la región axilar con material standard para disección, a través de un abordaje que comenzó en el piso de la axila removiendo la piel y fascia axilar, continuando con la disección del músculo pectoral mayor desde su inserción en el húmero, así como de la fascia clavipectoral (abordaje anteroinferior), para exponer el contenido de la fosa axilar. En algunas muestras la fosa axilar ya se encontraba expuesta, con el músculo pectoral mayor removido parcial o totalmente, así como el músculo pectoral menor y su fascia.

El tejido conectivo contenido en la fosa fue cuidadosamente removido para exponer lentamente los vasos axilares y el plexo braquial, hasta alcanzar al músculo subescapular y su fascia en la pared posterior de la fosa axilar, conservando el contenido neurovascular de la axila. Este procedimiento permitió ubicar varias referencias para encontrar rápidamente al fascículo posterior del plexo braquial (FPPB) y sus ramos tanto colaterales como terminales. Se identificó a los distintos nervios subescapulares siguiéndolos desde su punto de ingreso en el músculo subescapular hacia sus respectivos orígenes, y se determinó su lugar de procedencia.

En particular el nervio subescapular inferior fue identificado tras encontrar al músculo redondo mayor y observarse los ramos nerviosos que lo inervaban, a partir de los cuales se hizo el seguimiento hacia proximal para determinar si procedía efectivamente del fascículo posterior del plexo braquial, como señala la literatura clásica, o si su origen estaba sujeto a alguna variación, originándose desde el nervio axilar, o del nervio radial. Además, se determinó si éste era individual en su origen o procedía de un tronco común con otro nervio.

Los hallazgos fueron registrados fotográficamente con una cámara Fujifilm ${ }^{\circledR}$ modelo Finepix S de 12 mega pixeles, y fueron representados esquemáticamente en un cuaderno de apuntes. De las características propias del nervio subescapular inferior primero se cuantificó el número de ramos directos para el músculo subescapular y la forma en que se distribuían hacia el mismo y al músculo redondo mayor, estableciendo patrones de ramificación y finalmente estableciendo una propuesta para su clasificación.

\section{RESULTADOS}

Se observó primero la procedencia del nervio subescapular inferior y de sus distintos ramos, y se agruparon según su origen y sus ramificaciones. En 3 de los casos $(10 \%)$ el nervio subescapular inferior procedía de un tronco común con el nervio toracodorsal, siendo 2 del lado izquierdo (6,6\%) y 1 del derecho (3,3\%); en 27 casos $(90 \%)$ procedía como ramo colateral directo del nervio axilar, 15 del lado izquierdo (50\%) y 12 del derecho (40\%), como se muestra en la Tabla I. En ningún caso el origen fue directo del fascículo posterior del plexo braquial, como sugiere la literatura clásica, o del nervio radial. Además, se cuantificó el número de ramos colaterales directos desde el nervio subescapular inferior que aportaban a la inervación del músculo subescapular, y a su vez la cantidad de ramos terminales que aportaba cada uno. Se observó que los nervios subescapulares inferiores entregaron de 1 a 4 ramos para la inervación del músculo subescapular, y en todos los casos sólo 1 ramo para el músculo redondo mayor. 
Considerando la cantidad y distribución de los ramos colaterales directos del nervio subescapular inferior, se identificaron 5 patrones de ramificación hacia el músculo subescapular y redondo mayor, describiéndose como nervio subescapular inferior Tipo I, al que enviaba 1 ramo al músculo subescapular; Tipo II, al que emitía 2 ramos y así para el III y IV, que aportaban 3 y 4 ramos, respectivamente. El tipo $\mathrm{V}$ entregaba 1 ramo exclusivo al músculo mencionado.

La Tabla II, muestra número de casos y lateralidad, respecto a los tipos mencionados.

Los patrones Tipo I, II, III y IV consisten en un nervio subescapular inferior que entrega 1, 2, 3 y 4 ramos colaterales para el músculo subescapular, respectivamente, conservando todos ellos sólo 1 ramo para el músculo redondo mayor. Se observó en 7 casos el NSI Tipo I (23,3 \%), 2 del lado derecho y 5 del lado izquierdo (Fig. 1, A); 15 casos el NSI Tipo II (50\%), lado derecho y 9 del lado izquierdo (Fig. 1, B); 4 casos el NSI Tipo III (13,3 \%), 3 del lado derecho y sólo 1 del lado izquierdo (Fig. 1, C), y en tan sólo 2 casos se observó el NSI Tipo IV (6,7 \%), 1 del lado derecho y 1 del lado izquierdo (Fig. 2, A).

Por otro lado, el patrón Tipo V corresponde a un nervio subescapular inferior que se dirige únicamente al músculo subescapular, observándose un nervio independiente para el músculo redondo mayor, lo cual se encontró en sólo 2 casos; de éstos, el nervio tuvo origen proximal al origen

del nervio subescapular inferior (1 caso del lado derecho) también desde el nervio axilar, describiéndose como patrón Tipo Va (Fig. 2, B) y distal al origen del nervio subescapular inferior (1 caso del lado izquierdo), desde el fascículo posterior del plexo braquial, describiéndose como patrón Tipo Vb (Fig. 2, C).

Adicionalmente se observó que los distintos ramos del nervio subescapular inferior se dividieron en varios ramos terminales que penetran al músculo subescapular, encontrándose en un rango entre 1 y 8 ramos, con un promedio de 4 ramos terminales para el músculo, como se muestra en la Tabla III.

Tabla I. Origen del nervio subescapular inferior.

\begin{tabular}{lccc}
\hline Lado & N.axilar & Tronco Común & $\%$ \\
\hline Izquier do & 15 & 2 & 56,6 \\
Derecho & 12 & 1 & 43,4 \\
Total & 27 & 3 & 100 \\
\hline
\end{tabular}

Tabla II. Frecuencia de los patrones de ramificación del NSI.

\begin{tabular}{lcccc}
\hline Tipo NSI & $\begin{array}{c}\text { Lateralidad } \\
\text { Derecho }\end{array}$ & Izquierdo & Total & $\%$ \\
\hline Tipo I & 2 & 5 & 7 & 23,3 \\
Tipo II & 6 & 9 & 15 & 50,0 \\
Tipo III & 3 & 1 & 4 & 13,3 \\
Tipo IV & 1 & 1 & 2 & 6,7 \\
Tipo V & 1 & 1 & 2 & 6,7 \\
Total & 13 & 17 & 30 & 100 \\
\hline
\end{tabular}
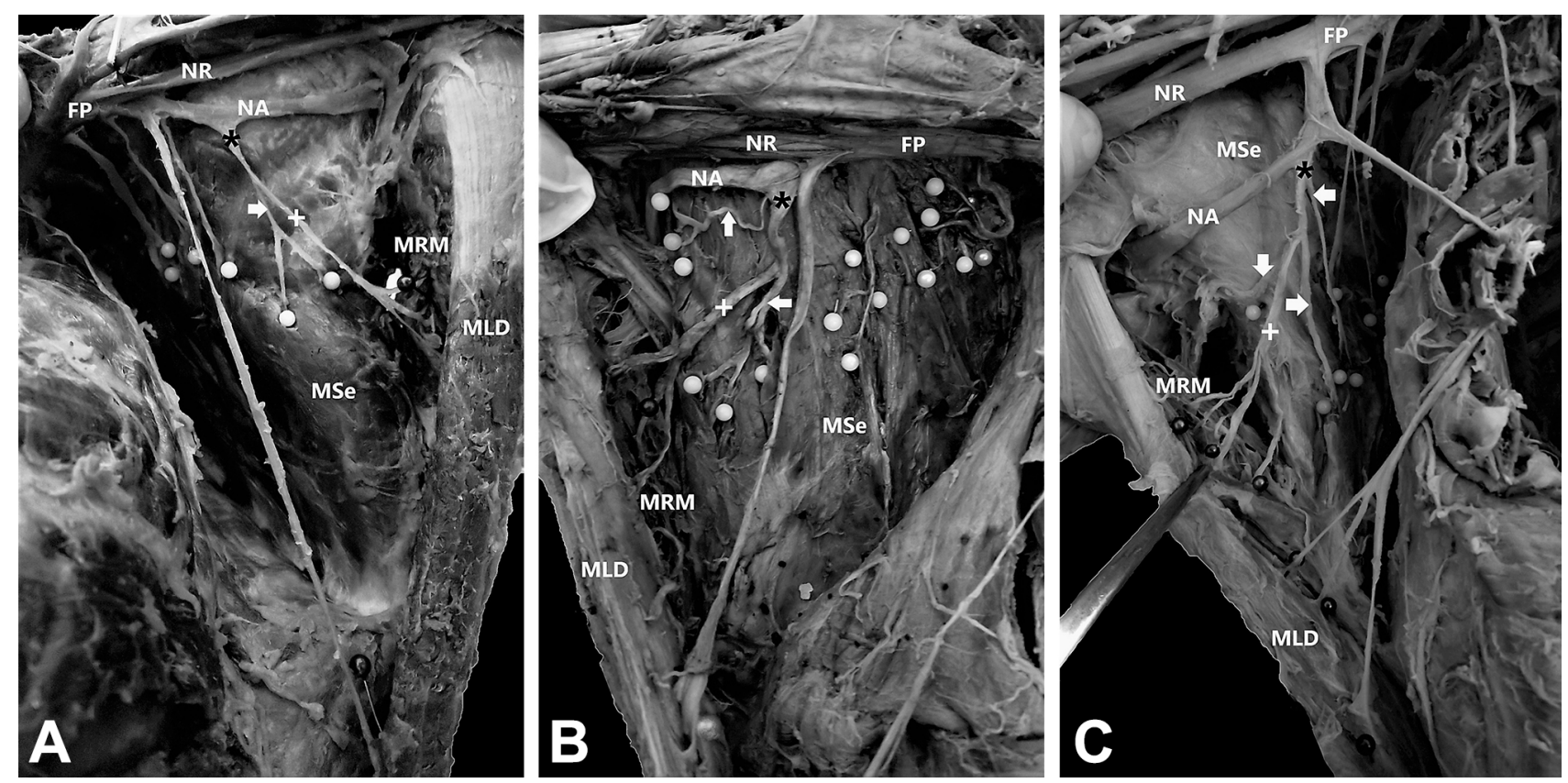

Fig. 1. Nervio Subescapular inferior tipo I (A), II (B) y III (C). FP, fascículo posterior; NR, nervio radial; NAx, nervio axilar; MSe, músculo subescapular; MRM, músculo redondo mayor; MLD, músculo latísimo del dorso; nervio subescapular inferior (*); ramos para el músculo subescapular (flechas); ramo para el músculo redondo mayor (+). 

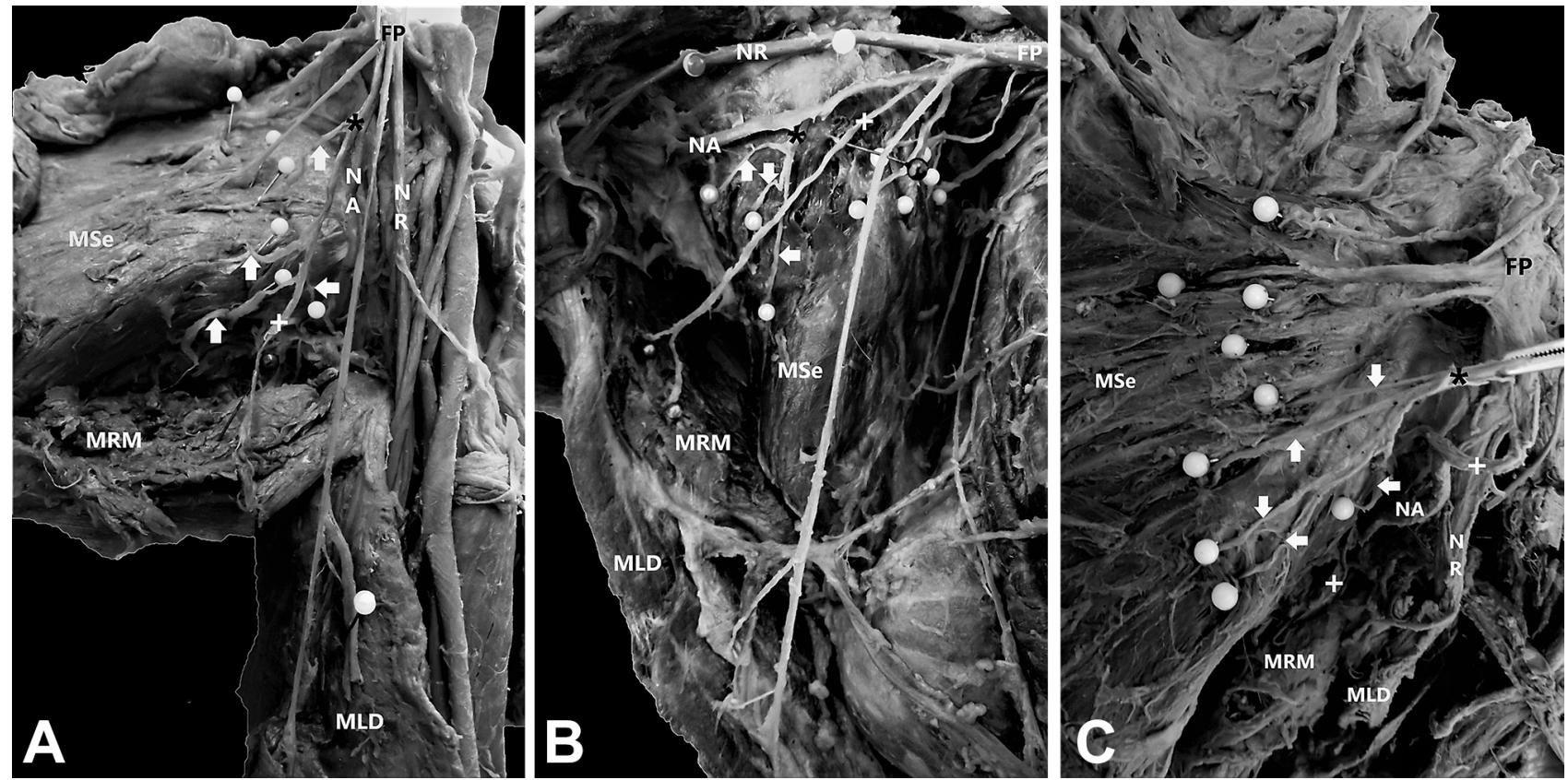

Fig. 2. Nervio Subescapular inferior tipo IV (A), Va (B) y Vb (C). FP, fascículo posterior; NR, nervio radial; NAx, nervio axilar; MSe, músculo subescapular; MRM, músculo redondo mayor; MLD, músculo latísimo del dorso; nervio subescapular inferior (*); ramos para el músculo subescapular (flechas); ramo para el músculo redondo mayor (+).

Tabla III. Número de ramos para el músculo subescapular.

\begin{tabular}{ccccc} 
Ramos & $\mathrm{N}^{\circ}$ Casos & Lado Izq. & Lado der. & $\%$ \\
1 & 1 & 1 & 0 & 3,3 \\
2 & 6 & 4 & 2 & 20 \\
3 & 9 & 5 & 4 & 30 \\
4 & 7 & 4 & 3 & 23,3 \\
5 & 2 & 2 & 0 & 6,7 \\
6 & 4 & 0 & 4 & 13,3 \\
8 & 1 & 1 & 0 & 3,3 \\
Total & 30 & 17 & 13 & 100 \\
\hline
\end{tabular}

\section{DISCUSIÓN}

El origen del nervio subescapular inferior se ha descrito clásicamente en la literatura como ramo directo del fascículo posterior del plexo braquial, con origen en los segmentos medulares C5 y C6 (Kim et al.). Sin embargo, la literatura actual lo sitúan con origen segmentario que es bastante variable, lo cual también se relaciona con el origen de este nervio, desde variadas porciones del plexo braquial (Aggarwal et al.; Kirik et al., 2017), hecho que también se puede corroborar con los resultados presentados en esta investigación.

En cuanto al origen del nervio subescapular inferior, el presente estudio mostró que fue con mayor frecuencia desde el nervio axilar, siendo así en un $90 \%$ de los casos, hallazgo que difiere a lo descrito por Kerr et al. (1918), que informaron un 54,14 \%, al $54 \%$ de Fazan et al. (2003), al
57,\% de Bhosale et al. (2014), 57,3\% de Muthoka et al. y que fue mencionado como una variación "frecuente" por Aggarwal et al., aunque no especificaron la cantidad. Al mismo tiempo, el resultado obtenido en el presente estudio es muy diferente de los descritos por McCann et al. (1994), donde el origen del nervio en cuestión, a partir del nervio axilar fue sólo en un $22 \%$ de los casos, similar a lo descrito por Tubbs et al. (2007), con $21 \%$, y por Chen et al. (2013), quienes lo reportó en un 16,7\%.

En estos mismos estudios, el origen del nervio subescapular inferior fue directo desde el fascículo posterior del plexo braquial en un $79 \%$ de los casos (Tubbs et al., 2007), en $39 \%$ (Fazan et al., 2003), en 37,5\% (Bhosale \& Havaldar), en 18,2\% (Chen et al.) y en $16 \%$ (Muthoka $e t$ al.), datos muy distintos a los del presente estudio, donde ningún caso mostró origen directo desde el fascículo posterior. Por otro lado, en un $10 \%$ de los casos el origen del nervio subescapular inferior procedía de un tronco común con el nervio toracodorsal, hecho que en la literatura se encontró poco documentado, salvo en lo descrito por Chen et al., quienes lo observaron en un 19,4\% de sus casos, Fazan et al. en un $9 \%$, Muthoka et al. en 1,3\%, además del reporte de un caso (Baliyan et al., 2011).

En casos que fueron una minoría, el nervio subescapular inferior se encontró "dividido", puesto que se encontró como ramo con destino exclusivo hacia el músculo subescapular, mientras que otro ramo independiente, con 
origen proximal o distal al nervio subescapular inferior, se dirigía hacia el músculo redondo mayor, hecho que no fue reportado en la literatura consultada, salvo en lo descrito en textos clásicos como Rouvière, donde se describe su recorrido anterior en relación al nervio subescapular, y una disposición terminal a través de varios ramos que abordan al músculo por su cara anterior, cerca de su inserción escapular, además de dirigirse también, en algunas ocasiones, a los fascículos inferiores y laterales del subescapular (Rouvière $\&$ Delmas).

Lo anterior, deja al descubierto un tema discutible, puesto que las características del nervio descrito como propio del nervio para el redondo mayor, son las mismas que otros autores definen para el nervio subescapular inferior, hecho que queda abierto a discusión y para investigaciones futuras.

Tener en cuenta las variaciones en el origen, cantidad y distribución del nervio subescapular inferior es importante para llevar a cabo con seguridad procedimientos quirúrgicos de la región axilar, como la artroplastia o reconstrucción de las estructuras de la articulación glenohumeral, considerando que el abordaje más común es el abordaje deltopectoral, en el cual se describe con frecuencia el compromiso funcional del músculo subescapular por daño de estructuras nerviosas (Walley et al., 2018).

En la contraparte, el bloqueo nervioso del músculo subescapular se describe como uno de los procedimientos más efectivos para el tratamiento del síndrome de "hombro congelado" (Jankovic \& van Zundert, 2006), para lo cual es fundamental tener en cuenta la variada contribución nerviosa hacia el músculo e identificar los principales troncos nerviosos que deben ser bloqueados. Además, síntomas de compresión nerviosa o de otras posibles patologías de la región axilar también pueden deberse a las variaciones descritas en este estudio (Ballesteros \& Ramirez).

Se ha documentado que el conocimiento de la distribución de ramos nerviosos puede ser un importante insumo para considerar procedimientos de transferencia nerviosa para la restitución de la inervación de la musculatura de la cintura escapular (Tubbs et al., 2006), o al mismo tiempo de procedimientos de neurectomía en caso de espasticidad (Mikalef \& Power, 2017). En relación a éste tema, la literatura cuenta con una amplia descripción de distintas técnicas de transferencia nerviosa para, por ejemplo, restituir de manera exitosa la abducción del hombro a través de la reinervación del músculo deltoides, utilizando principalmente ramos motores del tríceps braquial procedentes del nervio radial (Rinker, 2015), así como el uso de ramos motores del nervio interóseo posterior para restituir la función extensora en la mano (Tung et al., 2013), situaciones que se han resuelto gracias al acabado conocimiento de las variaciones en las estructuras intervenidas.

Pese a lo anterior, no se encontró evidencia de técnicas de transferencia nerviosa para el músculo subescapular, o desde algunos de los ramos nerviosos del músculo subescapular para la reinervación de otros grupos musculares del hombro, por lo que se considera que ésta investigación aporta conocimiento para considerar estudios sobre intervenciones de este tipo.

En la bibliografía actual tampoco fue posible encontrar una clasificación de la distribución de los ramos del nervio subescapular inferior en patrones de ramificación, al menos no en sujetos latinoamericanos. Lee et al. (2017), por ejemplo, describen y clasifican muy minuciosamente la localización de los principales ramos motores para 23 diferentes músculos del miembro superior, refiriéndose al patrón que existe en cada caso y la utilidad de conocerlo para el desarrollo de técnicas de transferencia tanto muscular como nerviosa, pero no incluye a ninguno de los músculos de la cintura escapular.

Las variaciones en el origen y distribución de los nervios del plexo braquial, en particular del nervio subescapular inferior, persisten como hallazgo frecuente tanto en procedimientos de disección de rutina como en la exploración quirúrgica de la zona axilar, por lo que al determinar su origen y distribución se espera contribuir con la actualización en el conocimiento de las amplias variaciones anatómicas del plexo braquial, para que junto a otros hallazgos, sean considerados antes de realizar tanto el diagnóstico como procedimientos de bloqueo nervioso o de intervención quirúrgica en la región cervical o axilar, así como también en la innovación para evitar lesiones iatrogénicas y perfeccionar las técnicas de transferencia nerviosa en la región escapular y axilar.

BUSTAMANTE ALISTE, P. A.; SOUSA RODRIGUES, C. F. \& OLAVE, E. Origin of the Inferior Subscapular nerve, branching patterns and its contribution to the subscapular muscle innervation. Int. J. Morphol., 38(1):176-181, 2020.

SUMMARY: The inferior subscapular nerve (ISN) partially innervates the subscapular muscle ( $\mathrm{SbM})$ and also innervates the teres major muscle (TMM). Several publications determine wide variation in their origin from Brachial Plexus (BP), but there is little evidence of these variations and the innervation pattern of SbM and TMMin Latin American individuals. The purpose of this study was to describe the origin of the ISN from $\mathrm{PB}$, to determine the number of branches that it gives to the SbM and the branching patterns. 30 upper limbs of cadavers of the Brazilian adult individuals were used; 13 on the right side and 17 
on the left, fixed in $10 \%$ formaldehyde. The axillary regions were dissected to expose the posterior fascicle of the brachial plexus (PFBP) and its branches. It was determined whether the origin of the NSI was individual or came from a common trunk. The number of branches for the SbM was quantified, establishing branching patterns. The ISN and its branches were grouped according to their origin and branching. In 3 of the cases (10\%) the ISN came from a common trunk with the thoracodorsal nerve (TDN), 2 from the left side $(6.6 \%)$ and 1 from the right side $(3.3 \%)$; in 27 cases $(90$ $\%)$ it came from the axillary nerve $(\mathrm{AxN}), 15$ from the left side (50 $\%)$ and 12 from the right side (40\%). In no case, the origin was direct from the PFBP. In addition, the number of branches that contributed to the innervation of the $\mathrm{SbM}$ was quantified, with an average of 4 branches (from 1 to 8 branches) being observed for the SbM. Four branching patterns of the ISN towards the SbM and the TMM were identified. Both the origin and the distribution of the ISN presented many variations. The data provided will complement the knowledge for proper teaching, timely diagnosis and good surgical practice of the axillary area.

KEY WORDS: Anatomy; Brachial Plexus; Inferior subescapular nerve; Subescapular muscle.

\section{REFERENCIA BIBLIOGRÁFICAS}

Aggarwal, A.; Harjeet, K.; Sahni, D. \& Aggarwal, A. Bilateral multiple complex variations in the formation and branching pattern of brachial plexus. Surg. Radiol. Anat., 31(9):723-31, 2009.

Baliyan, R.; Mehta, V.; Arora, J.; Nayyar, A. K.; Suri, R. K. \& Rath, G. Unilateral intercordal neural communication coexistent with variant branching pattern of posterior cord of brachial plexus. Acta Medica (Hradec Kralove), 54(3):131-4, 2011.

Ballesteros, L. E. \& Ramirez, L. M. Variations of the origin of collateral branches emerging from the posterior aspect of the brachial plexus. $J$. Brachial Plex. Peripher. Nerve Inj., 2:14, 2007.

Bhosale, S. M. \& Havaldar, P. P. Study of variations in the branching pattern of lower subscapular nerve. J. Clin. Diagn. Res., 8(11):AC05-7, 2014.

Chen, L. Z.; Chen, L.; Zhu, Y. \& Gu, Y. D. Semiquantifying of fascicles of the $\mathrm{C} 7$ spinal nerve in the upper and lower subscapular nerves innervating the subscapularis and its clinical inference in Erb's palsy. Clin. Anat., 26(4):470-5, 2013.

Dancker, M.; Lambert, S. \& Brenner, E. The neurovascular anatomy of the teres major muscle. J. Shoulder Elbow Surg., 24(3):e57-67, 2015.

Fazan, V. P. S.; Amadeu, A. de S.; Caleffi, A. L. \& Rodrigues Filho, O. A. Brachial plexus variations in its formation and main branches. Acta Cir. Bras., 18 Suppl. 5:14-8, 2003.

Federative Committee on Anatomical Terminology (FCAT). Terminologia Anatomica: International Anatomical Terminology. Stuttgart, Thieme, 1998. pp. 138

Jankovic, D. \& van Zundert, A. The frozen shoulder syndrome. Description of a new technique and five case reports using the subscapular nerve block and subscapularis trigger point infiltration. Acta Anaesth. Belg., 57(2):137-43, 2006.

Kerr, A. T. The brachial plexus of nerves in man, the variations in its formation and branches. Am. J. Anat., 23(2):285-395, 1918.

Kim, D. I.; Lee, K. S.; Kim, H. J.; Chae, H. K. \& Yu, K. H. The spinal root origins and clinical implications of the lower subscapular nerve. Clin. Anat., 21(5):416-9, 2008.

Kirik, A.; Mut, S. E.; Daneyemez, M. K. \& Seçer, H. I'. Anatomical variations of brachial plexus in fetal cadavers. Turk. Neurosurg., 2017.
In Press. doi: 10.5137/1019-5149.JTN.21339-17.2

Lee, E. Y.; Sebastin, S. J.; Cheah, A.; Kumar, V. P. \& Lim, A. Y. T. Upper extremity innervation patterns and clinical implications for nerve and tendon transfer. Plast. Reconstr. Surg., 140(6):1209-19, 2017.

McCann, P. D.; Cordasco, F. A.; Ticker, J. B.; Kadaba, M. P.; Wootten, M. E.; April, E. W. \& Bigliani, L. U. An anatomic study of the subscapular nerves: A guide for electromyographic analysis of the subscapularis muscle. J. Shoulder Elbow Surg., 3(2):94-9, 1994.

Mikalef, P. \& Power, D. The role of neurectomy in the management of spasticity of the upper limb. EFORT Open Rev., 2(11):469-73, 2017.

Moore, K.; Dailey, A. \& Agur, A. Anatomía con Orientación Clínica. $7^{\mathrm{a}}$ ed. Barcelona, Wolters Kluwer Health, Lippincot Williams \& Wilkins, 2013.

Muthoka, J. M.; Sinkeet, S. R.; Shahbal, S. H.; Matakwa, L. C. \& Ogeng'o, J. A. Variations in branching of the posterior cord of brachial plexus in a Kenyan population. J. Brachial Plex. Peripher. Nerve Inj., 6:1, 2011.

Rinker, B. Nerve transfers in the upper extremity: a practical user's guide. Ann. Plast. Surg., 74 Suppl. 4:S222-8, 2015.

Rouviére, H. \& Delmas, A. Anatomía Humana. Descriptiva, Topográfica y Funcional. Tomo 3 Miembros. $11^{\mathrm{a}}$ ed. Barcelona, Masson, 2005.

Tubbs, R. S.; Khoury, C. A.; Salter, E. G.; Acakpo-Satchivi, L.; Wellons, J. C. 3rd.; Blount, J. P. \& Oakes, W. J. Quantitation of the lower subscapular nerve for potential use in neurotization procedures. $J$. Neurosurg., 105(6):881-3, 2006.

Tubbs, R. S.; Loukas, M.; Shahid, K.; Judge, T.; Pinyard, J.; Shoja, M. M.; Slappey, J. B.; McEvoy, W. C. \& Oakes, W. J.. Anatomy and quantitation of the subscapular nerves. Clin. Anat., 20(6):656-9, 2007.

Tung, T. H.; Barbour, J. R.; Gontre, G.; Daliwal, G. \& Mackinnon, S. E. Transfer of the extensor digiti minimi and extensor carpi ulnaris branches of the posterior interosseous nerve to restore intrinsic hand function: case report and anatomic study. J. Hand Surg. Am., 38(1):98103, 2013.

Uzun, A. \& Bilgiç, S. Some variations in the formation of the brachial plexus in infants. Turk. J. Med. Sci., 20:573-57, 1999.

Walley, K. C.; Saloky, K. L.; Updegrove, G. F.; Lopez, H. \& Armstrong, A. D. Characterization of an anatomic safe zone surrounding the lower subscapular nerve during an open deltopectoral approach. J. Shoulder Elbow Surg., 28(4):671-7, 2018.

\section{Dirección para correspondencia:}

Dr. Enrique Olave

Facultad de Medicina

Universidad de La Frontera

Av. Francisco Salazar 01145

Temuco

CHILE

Email: enrique.olave@ufrontera.cl

Recibido : 15-08-2019

Aceptado: 20-09-2019 